\title{
Research on Data Fitting Model Based on Positioning of Digital Zenith Camera
}

\author{
Shang Yang ${ }^{\mathrm{a}}$,Zhaofa Zhou ${ }^{\mathrm{b}}$,Xianyi Liu ${ }^{\mathrm{c}}$ \\ Xi'an Institute of High-Tech, Hongqing Town, Xi'an, Shanxi, 710025, PR China. \\ a1306676498@qq.com, bm18592057570@163.com,cysjiayouys@163.com
}

\begin{abstract}
The data fitting model is the basis for data processing in the positioning of digital zenith camera. In this paper, the similarity transformation model, affine transformation model, projective transformation model and the solution of parameters of these models were strictly deduced. And the three data fitting models were applied to the positioning algorithm of the digital zenith camera. Experimental results showed:When the similarity transformation model and the affine transformation model were used for positioning, the positioning accuracy of the digital zenith camera was basically the same. When the projection transformation model were used for positioning, the accuracy of the data fitting and the positioning accuracy of the digital zenith instrument were better than those of the other two data fitting models.
\end{abstract}

Keywords: Data Fitting Model,Astronomical Positioning,Digital Zenith camera.

\section{Introduction}

The digital zenith camera is a high-precision astronomical positioning instrument that can achieve automation, high efficiency, high-precision positioning, and is widely used in the field of geodetic measurement(Hirt C, Bürki B,et al,2010). The digital zenith camera is a sophisticated and sophisticated instrument consisting of a digital zenith camera, a rotating platform, a control system, a punctuality system, a data processing system, and auxiliary equipment. The successful development of the digital zenith indicates that the earth astronomical measurement has entered the digital age(Lili TIAN, Jinyun GUO,et al,2014). The digital zenith camera photographs star of the zenith direction by means of rotation shooting, and uses the star map data identified and the time information provided by the GPS to establish a fitting relationship between the CCD image coordinates and the celestial tangent plane coordinate. On this basis, The astronomical longitude and latitude of the rotating axis of the digital zenith camera are obtained by iterative calculation(Bo WANG,Lili TIAN,et al,2014). Establishing a data fitting model between CCD image coordinates and tangent plane coordinates is a key link in the positioning process of digital zenith camera. This not only relates to the accuracy of the data fitting, but also relates to the positioning accuracy of the digital zenith camera. The more accurate the data fitting model, the higher the positioning accuracy of the digital zenith camera, so choosing an appropriate data fitting model is of great significance for carrying out research on the method of digital zenith camera positioning.

At present, the positioning of digital zenith cameras developed at home and abroad is mainly based on the state of precision leveling. Even with a fine leveling, there is still a small angle between the CCD plane and the tangent plane of the digital Zenith camera(Xihui ZHANG, Zhaofa ZHOU,et al, 2018). For this reason, this paper deduces the similarity transformation model and the radiation transformation model without considering the existence of the small dip angle, and deduces the projection transformation model with considering the existence of the small dip angle. the effect of different data fitting models on the positioning accuracy of the digital zenith is analyzed through experiments.

\section{The Establishment of Data Fitting Model}

\subsection{Establishment of Similarity Transformation Model}

During the positioning process of the digital zenith camera, it is necessary to realize the conversion between the CCD image coordinates and the celestial tangent plane coordinates. For this purpose, the 
CCD image coordinate system and the tangent plane coordinate system are introduced. As shown in Figure 1, $O-\xi \eta$ is the tangent plane coordinate system. The origin of the tangent plane coordinate system is the intersection of the rotation axis of the digital zenith and the celestial sphere. The $O \xi$ axis and the $O \eta$ axis point to the east and the north. $O-x y$ is the CCD image coordinate system, its coordinate origin is in the center of the CCD sensor, and the coordinate axes of CCD image coordinate system are in the same direction as the two sides along the CCD sensor.

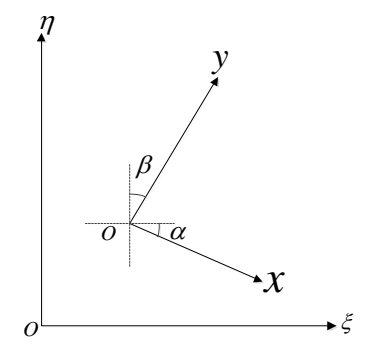

Fig 1.Data Fitting Model

For the similar transformation model, the two coordinate axes of the CCD image coordinate system are strictly orthogonal, that is $\alpha=\beta$, the scale coefficient of the two coordinate axes is 1 , according to the principle of coordinate transformation.(QIN Yongyaun,2014):

Simplify the above formula available:

$$
\left[\begin{array}{l}
\eta \\
\xi
\end{array}\right]=\left[\begin{array}{cc}
-\sin \alpha & \cos \alpha \\
\cos \alpha & \sin \alpha
\end{array}\right]\left[\begin{array}{l}
x \\
y
\end{array}\right]+\left[\begin{array}{l}
c_{1} \\
c_{2}
\end{array}\right]
$$

$$
\left\{\begin{array}{l}
\eta=-x \sin \alpha+y \cos \alpha+c_{2} \\
\xi=x \cos \alpha+y \sin \alpha+c_{1}
\end{array}\right.
$$

Let $a=\sin \alpha, b=\cos \alpha$,Simplify the above formula to get:

$$
\left\{\begin{array}{l}
\eta=-a x+b y+c_{1} \\
\xi=b x+a y+c_{2}
\end{array}\right.
$$

The above equation is a similar transformation model.

\subsection{The Establishment of Affine Transformation Model}

Under external interference conditions, such as temperature changes, installation errors, etc., which cause slight deformation of the CCD sensor, the two coordinate axes of the CCD image coordinate system established on the basis of the CCD sensor are not strictly orthogonal and the scale coefficient between the two axes is not the same. In this regard, this paper assumes that the angle between the $O y$ axis and the $O \eta$ axis is $\beta$, the angle between the $O x$ axis and the $O \xi$ axis is $\alpha$, and the scale coefficient of the two axes is $m, n$, then there exists:

$$
\left[\begin{array}{l}
\eta \\
\xi
\end{array}\right]=\left[\begin{array}{cc}
-\sin \beta & \cos \beta \\
\cos \alpha & \sin \alpha
\end{array}\right]\left[\begin{array}{l}
m x \\
n y
\end{array}\right]+\left[\begin{array}{l}
c_{1} \\
c_{2}
\end{array}\right]
$$

Simplify the above formula to get:

$$
\left\{\begin{array}{l}
\eta=-m x \sin \beta+n y \cos \beta+c_{2} \\
\xi=m x \cos \alpha+n y \sin \alpha+c_{1}
\end{array}\right.
$$

Let $a_{1}=-m \sin \beta, b_{1}=n \sin \beta, a_{2}=m \cos \alpha, b_{2}=n \sin \alpha$, Simplify the above formula to get:

$$
\left\{\begin{array}{l}
\eta=a_{1} x+b_{1} y+c_{1} \\
\xi=a_{2} x+b_{2} y+c_{2}
\end{array}\right.
$$

The above equation is an affine transformation model.

\subsection{The Establishment of Projection Transformation Model}

The first two models assume that the CCD plane and the tangent plane are parallel to each other. While in the actual shooting state, there is an angle within $\pm 10^{\prime \prime}$ degrees between the CCD plane and the star tangent plane(Xihui ZHANG, Zhaofa ZHOU,et al, 2018). In this regard, the projection 
transformation model is established in this paper to accurately reflect the mapping relationship between the CCD image coordinate system and the tangent plane quasi coordinate system to improve the accuracy of the data fitting model. As shown in Fig.2, the dashed line frame is a CCD plane in an inclined state, and the solid line frame is a horizontal plane. The optical axis $\mathrm{X}$ is always perpendicular to the CCD plane and passes over the center of the CCD sensor. Point $A$ is the intersection of the optical axis and the CCD plane.,Point $O$ is the intersection of the optical axis and the horizontal plane. Point $B, D$ is the point of stellar imaging on the CCD plane and imaging on the horizontal plane. Point $E$ is the projection of the focus on the horizontal plane. First, establish the CCD image coordinate system $\mathrm{O}-x y z$, whose origin is the intersection of the optical axis of the digital zenith camera and the CCD plane. Then use the horizontal plane as a reference to establish a tangent plane coordinate system. The origin of the tangent plane coordinate system coincides with the origin of the $\mathrm{CCD}$ image coordinate system. The $\mathrm{X}$ axis points to the east, the $\mathrm{Y}$ axis points to the north, and the $\mathrm{Z}$ axis points to the direction of the zenith; Finally, establish a spatial auxiliary coordinate system $O^{\prime}-u v w$, The spatial auxiliary coordinate system is parallel to the CCD north reference coordinate system, In the spatial auxiliary coordinate system, Assume that the coordinate of point $E$ is $\left(u_{E}, v_{E}, w_{E}\right)$, The coordinate of point $\mathrm{A}$ is $\left(u_{A}, v_{A}, w_{A}\right)$, There are $B E=u_{A}-u_{E}, A B=v_{A}-v_{E}$, In the tangent plane coordinate system, assume $C D=X, D O=Y, C F=Z$.

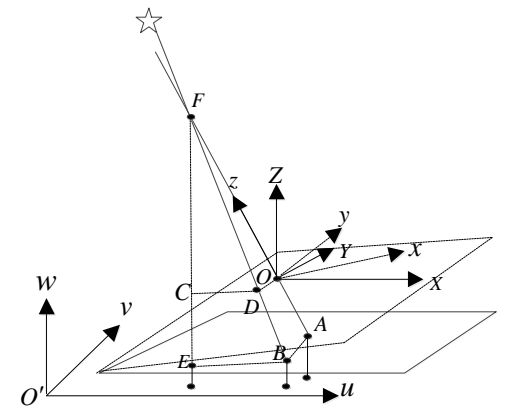

Fig 2.Coordinate transformation model

The figure 2 are analyzed to get:

$$
\frac{X}{u_{A}-u_{E}}=\frac{Y}{v_{A}-v_{E}}=\frac{Z}{w_{F}-w_{A}}
$$

In the CCD image coordinate system, suppose there is a point with a coordinate of $(x, y,-f)$, Then there is a coordinate transformation matrix $\boldsymbol{R}$, which can convert the coordinates from the CCD image coordinate system to the spatial auxiliary coordinate system. There is

$$
\left[\begin{array}{l}
u \\
v \\
w
\end{array}\right]=\boldsymbol{R}\left[\begin{array}{c}
x \\
y \\
-f
\end{array}\right]=\left[\begin{array}{lll}
a_{1} & a_{2} & a_{3} \\
b_{1} & b_{2} & b_{3} \\
c_{1} & c_{2} & c_{3}
\end{array}\right]\left[\begin{array}{c}
x \\
y \\
-f
\end{array}\right]
$$

In conjunction with (7) and (8), There is

$$
\left\{\begin{array}{l}
x=-f \frac{a_{1}\left(u_{A}-u_{E}\right)+b_{1}\left(v_{A}-v_{E}\right)+c_{1}\left(w_{F}-w_{A}\right)}{a_{3}\left(u_{A}-u_{E}\right)+b_{3}\left(v_{A}-v_{E}\right)+c_{3}\left(w_{F}-w_{A}\right)} \\
y=-f \frac{a_{2}\left(u_{A}-u_{E}\right)+b_{2}\left(v_{A}-v_{E}\right)+c_{2}\left(w_{F}-w_{A}\right)}{a_{3}\left(u_{A}-u_{E}\right)+b_{3}\left(v_{A}-v_{E}\right)+c_{3}\left(w_{F}-w_{A}\right)}
\end{array}\right.
$$

Since coordinate system $O-X Y Z$ is parallel to coordinate system $O-u v w$, there is $w_{A}-w_{F}=H$, Since $u_{A}-u_{S}=X, v_{A}-v_{S}=Y$, equation (9) can be simplified as:

Equation (10) can be simplified as:

$$
\left\{\begin{array}{l}
X_{A}-X_{S}=H \frac{a_{1} x+a_{2} y-a_{3} f}{c_{1} x+c_{2} y-c_{3} f} \\
Y_{A}-Y_{S}=H \frac{b_{1} x+b_{2} y-b_{3} f}{c_{1} x+c_{2} y-c_{3} f}
\end{array}\right.
$$




$$
\left\{\begin{array}{l}
X=\frac{A_{1} x+B_{1} y+C_{1}}{1+D x+E y} \\
Y=\frac{A_{2} x+B_{2} y+C_{2}}{1+D x+E y}
\end{array}\right.
$$

The formula (11) is a projection transformation model. there is $A_{1}=-\frac{a_{1} H}{c_{3} f}, B_{1}=-\frac{a_{2} H}{c_{3} f}, C_{1}=\frac{a_{3} H}{c_{3}}$, $A_{2}=-\frac{b_{1} H}{c_{3} f}, B_{2}=-\frac{b_{2} H}{c_{3} f}, C_{2}=\frac{b_{3} H}{c_{3}}, D=-\frac{c_{1}}{c_{3} f}, E=-\frac{c_{2}}{c_{3} f}$. Considering the tilt between the two coordinate planes, the coefficient $D, E$ was introduced to make the data fitting more accurate.

\section{Analysis of Experimental Data}

In order to analyze the different data fitting models for the analysis of the positioning results of the digital zenith camera, a digital zenith camera was used for positioning experiments. The effective visual field angle of the digital zenith instrument used in the experiment is $3^{\circ} \times 3^{\circ}$. The CCD sensor is a KAF-16803 area array CCD produced by Kodak Corporation. The size of the CCD sensor is 4096 pixel $\times 4096$ pixel and the single pixel size is $9 \mu \mathrm{m} \times 9 \mu \mathrm{m}$. Table 1 shows the results of star identification of the digital zenith camera.

Table 1.Results of star identification

\begin{tabular}{ccccc}
\hline CCDx coordinate & CCDy coordinate & Star longitude & Star latitude & Star number \\
\hline 3867.242 & 2744.653 & 107.994 & 32.918 & 54134 \\
101.965 & 2653.682 & 111.197 & 34.766 & 55143 \\
1579.506 & 1604.052 & 109.300 & 34.830 & 54548 \\
721.474 & 2575.566 & 110.608 & 34.535 & 54936 \\
2078.863 & 1361.137 & 108.724 & 34.766 & 54369 \\
3854.523 & 2954.750 & 108.125 & 32.775 & 54183 \\
2130.045 & 2082.409 & 109.098 & 34.225 & 54491 \\
2458.066 & 2465.764 & 109.036 & 33.794 & 54471 \\
\hline
\end{tabular}

In order to analyze the accuracy of different data fitting models, the distribution of the residual values was analyzed after the three data fitting models were used to fit the CCD image coordinates and the tangent plane coordinates. The results are shown in the figure 3.

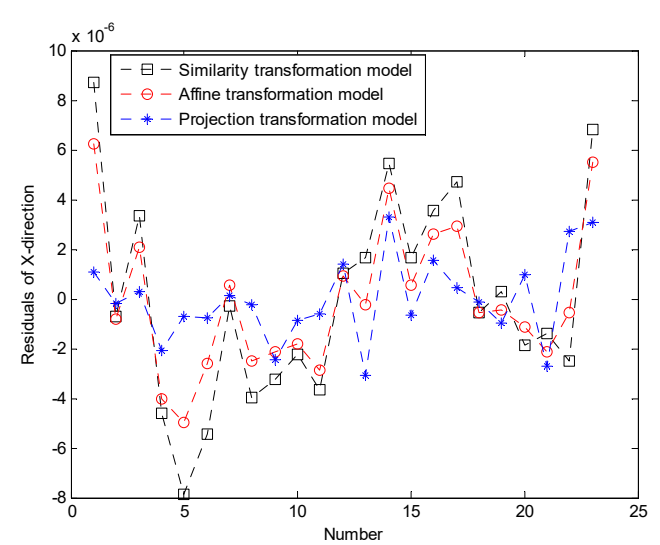

a.Residuals of X-direction

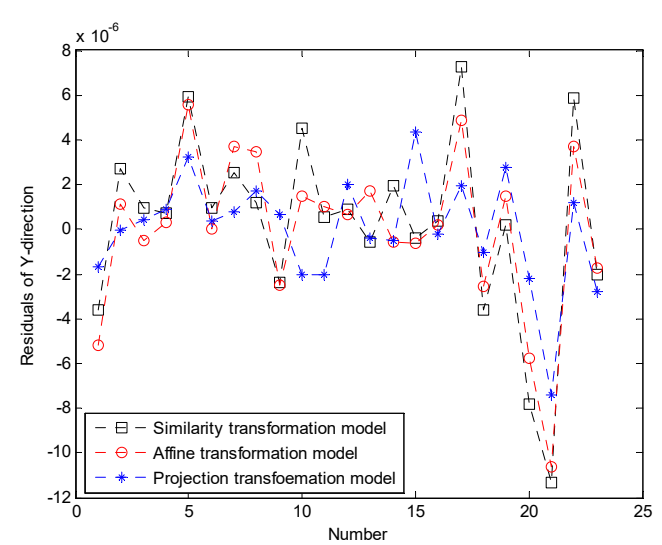

b.Residuals of Y-direction

Fig 3.Residual distribution

From the analysis of Figure 3, the distribution of the residual values of the similarity transformation model is the most dispersed. The standard deviations of the residuals in X-direction and Y-direction are $4.21 \times 10^{-6}$ and $2.89 \times 10^{-6}$. The residual distribution of the affine transformation model is more centralized than the similarity transformation model. The standard deviations of the 
residuals in X-direction and Y-direction are $3.65 \times 10^{-6}$ and $2.84 \times 10^{-6}$, The residual distribution of projection transformation model is the most concentrated, The standard deviation of the residuals in $\mathrm{X}$-direction and Y-direction are $2.44 \times 10^{-6}$ and $2.01 \times 10^{-6}$, So the projection transformation model has the highest accuracy for data fitting.

Three models were used for positioning. The positioning result of the digital zenith camera is shown in Fig.4.
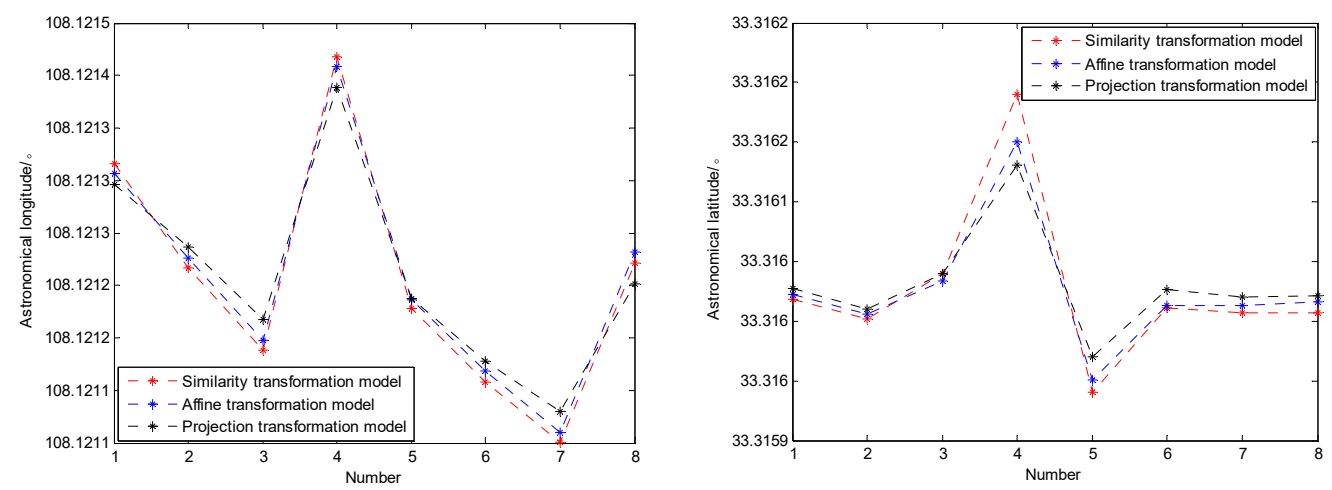

Fig 4.Positioning results

From the analysis of Figure 4, the positioning accuracy of the digital zenith is basically the same with the similar transformation model and the affine transformation model for positioning. Compared with similar transformation model and affine transformation model, projection transformation model is used for positioning, and the digital zenith camera has the highest positioning accuracy. Among them, the similarity transformation model is used for positioning. The median error of astronomical longitude is $0.42^{\prime \prime}$, the median error of the astronomical latitude is $0.35^{\prime \prime}$. The affine transformation model is used for positioning. The medium error of astronomical longitude is $0.40^{\prime \prime}$, and the medium error of astronomical latitude is $0.31^{\prime \prime}$, the projective transformation model is used for positioning. The median error of astronomical longitude is $0.34 "$, and the median error of astronomical latitude is $0.23^{\prime \prime}$.

\section{Summary}

Through the analysis of different data fitting models, since the projection transformation model takes into account the small inclination between the CCD plane and the tangent plane, the accuracy for the data fitting is the highest with the projection transformation model. Comparing with the similarity transformation model and the projection transformation model, the digital zenith camera has the highest positioning accuracy with the projection transformation model for positioning.

\section{References}

[1]. Hirt C Bürki B,Guillaume S,et al.Digital zenith cameras-state-of-the-art astrogeodetic technology for Australian Geodesy[C].FIG Congress-Remote Sensing and Optical Techniques I,Sydney:Department of Land Management.2010:1-16.

[2]. Lili TIAN, Jinyun GUO, Yanben HAN,et al.Digital zenith telescope prototype of China[J].Chin.Sci.Bull,2014,59(12):1094-1099.

[3]. Bo WANG,Lili TIAN,Zheng WANG,et al.The image and data processing in digital zenith camera[J]. Chin.Sci.Bull,2014,59(12):1100-1107.

[4]. Xihui ZHANG, Zhaofa ZHOU, Xianyi LIU, et al. Analysis of the fast positioning method of digaital zenith camera in tilt state[J]. Infrared and Laser Engineering, 2018, 47(2):0217002102170026.

[5]. QIN Yongyaun.Inertial Navigation[M].Beijing:Science Press,2014:244-252. 
[6]. Xianyi LIU,Zhaofa ZHOU,Zhili ZHANG,et al.Research on the Transformation of Coordinates Astronomical Fixation[J].Electronics Optics\&Control,2016,23(1)11-14. 